\title{
УДК 553.984 \\ ОПРЕДЕЛЕНИЕ И ХАРАКТЕРИСТИКА КОЭФФИЦИЕНТА СВЯЗНОСТИ КОЛЛЕКТОРА ГЕОЛОГО-ГИДРОДИНАМИЧЕСКИХ МОДЕЛЕЙ НЕФТЕГАЗОВЫХ ЗАЛЕЖЕЙ
}

\author{
Попов Виктор Львович1, \\ Popovvl@tomsknipi.ru
Поднебесных Александр Владимирович2, PodnebesnykhAV@mail.ru \\ Пыльник Сергей Валерьевич1, \\ PylnikSV@tomsknipi.ru \\ 1 ОАО «ТомскНИПИнефть», \\ Россия, 635026, г. Томск, пр. Мира, 72. \\ 2 Уфимский государственный нефртяной технический университет, \\ Россия, 450062, г. Уфра, ул. Космонавтов, 1.
}

\begin{abstract}
Актуальность исследования обусловлена необходимостью количественной оценки связности коллектора геологогидродинамических моделей для классификации реализаций при многовариантной оценке неопределенностей и оптимизации выбора метода и параметров моделирования куба песчанистости.

Цель: ввести понятие коэффициента связности коллектора, оценить репрезентативность предложенного параметра для моделей залежей различной детальности и геометрии; оценка влияния параметров неоднородности геологических моделей на коэфффициент связности.

Объекты: геолого-гидродинамические модели залежей нефтегазовых месторождений.

Методы: геологическое и гидродинамическое моделирование, статистический анализ результатов геологического и гидродинамического моделирования.

Результаты. Кратко рассмотрены существующие методы оценки связности коллектора в геолого-гидродинамических моделях, используемые для оценки ресурсов углеводородного сырья и выбора наиболее эфффективных методов их разработки. На основе проведенного авторами анализа был предложен новый подход к определению параметра связанности как отношения перетока жидкости в исследуемой модели к модели с единичной песчанистостью. Рассмотрено влияние геометрии и детальности геолого-гидродинамических моделей на величину коэфффициент связности. На примере синтетических стохастических моделей рассмотрены зависимости коэффрициента связности от таких параметров, как песчанистость, ранги вариограмм, их анизотропия и эфффект самородка. Введено понятие коэфффициента гидродинамической связности коллектора, который характеризует способность модели фрильтровать фрлюид, без учета влияния фрильтрационно-емкостных характеристик. Этот параметр учитывает только распределение коллектора в объеме залежи и то, как ячейки коллектора расположены друг относительно друга. Предложенный способ расчёта позволяет оценить анизотропию перетоков фрлюида между ячейками по различным направлениям и оценить способность модели фильттровать этот фрлюид через себя. Коэфрфициент связанности, в отличие от перколяционных методов, позволяет получать непрерывную оценку гидродинамической связи между ячейками, не требует обязательного наличия истории работы залежи и не зависит от текущей системы разработки, реализуемой на месторождении.
\end{abstract}

\section{Ключевые слова:}

Геологическая модель, связность коллектора, коэфффициент связности, вариограмма, эфффект самородков, последовательное индикаторное моделирование, теория перколяции, многоскважинный индекс продуктивности.

\section{Существующие методы оценки связности коллектора}

В настоящее время цифровые геологогидродинамические модели используются для оценки величины запасов углеводородов (УВ) и для проектирования разработки залежей нефти и газа. Одним из важнейших параметров геолого-гидродинамических моделей, влияющих на распределение запасов в объеме резервуара, является неоднородность коллектора и его гидродинамическая связность $[1,2]$.

Обзор опубликованной литературы показал, что до сих пор нет устоявшегося определения связности и методов его количественного выражения [3-11]. Одни из них основаны на теории перколяции, и их условно можно назвать методами оценки геометрической связности $[8,9]$, другие базируются на анализе данных добычи и закачки скважин на месторождении, их принято считать методами оценки гидродинамической связности $[6,10]$.

Теория перколяции (протекания) - сравнительно молодая отрасль науки, занимающаяся изучением критических явлений в стохастических неоднородных средах, таких как протекание жидкости через пористую среду, намагничивание ферромагнетиков, протекание тока в полупроводниках и т. п. [11]

Классическим примером объекта исследований теории перколяции может служить опыт Ватсона и Лиса [12]. Суть его заключалась в том, что исследовалась электрическая проводимость между двумя электродами, соединенными проволочной сеткой. В сетке случайным образом выбирался узел, в котором контакты рвались, далее также случайным образом выбирался следующий и т. д. (рис. 1). В тот мо- 
мент, когда рвалось последнее соединение между электродами, происходило скачкообразное изменение состояния системы из проводящего в непроводящее.

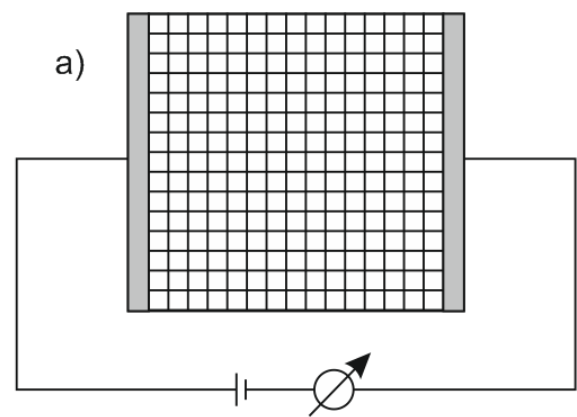

b)

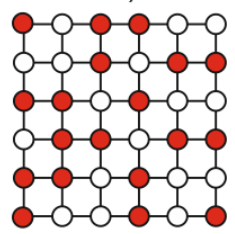

c)

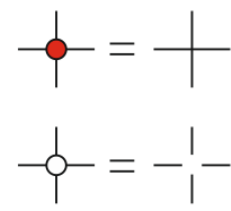

Pис. 1. Схема эксперимента Ватсона и Лиса: а) исходная сетка. Количество узлов на рисунке сильно уменьшено; b) кусок сетки с блокированными (красные) и неблокированными (белыми) узлами; c) светлый узел означает разрыв контакта между четырьмя проволоками, которые связывают узел, красный узел сохраняет контакт [12]

Fig. 1. Scheme of the Watson and Liss experiment: a) initial grid. The number of nodes is lowered; b) blocked nodes are red circles, unblocked ones are white; c) light node means loss of contact between four ends. Red contact means four ends are connected. The current cannot flow through light node in any direction and can flow through red node in any direction

Подобную по строению и функционированию систему представляет собой и геолого-гидродинамическая модель, в которой роль узлов-проводников выполняют ячейки коллектора, роль блокированных узлов ячейки неколлектора, а нахождение пределов и условий для протекания жидкости через модель и является предметом изучения теории перколяции [13].

На основе теории перколяции авторами [10] были предложены новые способы оценки связности геологических моделей, такие как функция связности зависимость вероятности наличия перколяционной связи между двумя ячейками от расстояния между этими ячейками (рис. 2).

Дж. Ховадик и Д. Лару [8] предложили другой способ оценки геометрической связности, основанный на применении теории перколяции к моделям залежей нефти и газа. Так, согласно этим авторам, связность коллектора можно количественно оценить как отношение объема самого большого кластера в модели к общему объему всех кластеров. Для планирования бурения и построения карт неоднородности свойств необходимо иметь возможность оценить связность локально. Для этих целей вышеуказанными авторами был предложен параметр, рассчитываю- щийся как отношение объема кластера, связанного со скважиной, к общему объему всех кластеров в модели.

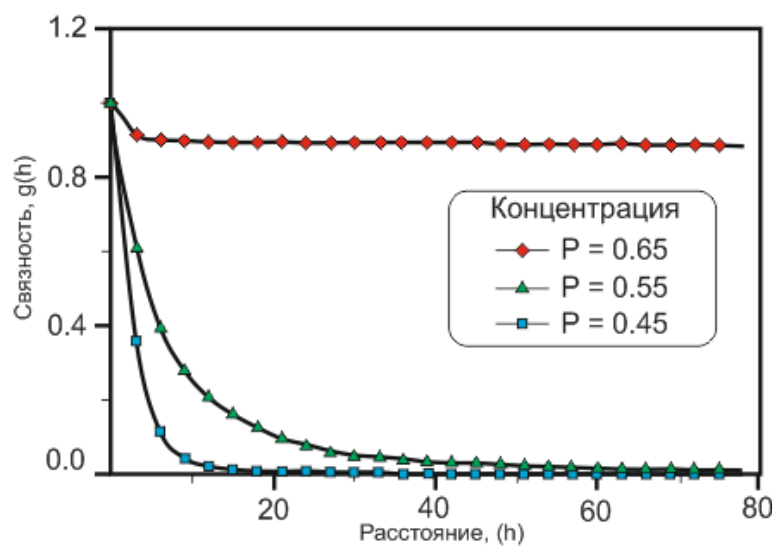

Pис. 2. Пример функиии связности для моделей с различным долями коллектора [10]

Fig. 2. Example of connectivity function for models with different concentrations [10]

Такие оценки связности геологических моделей, основанные на теории перколяции, обладают двумя существенными недостатками. Во-первых, предполагается, что процесс фильтрации флюида будет иметь критический характер, т. е. между двумя точками связность можно оценить двумя состояниями: связность присутствует и связность отсутствует. Переход между этими состояниями происходит скачкообразно при небольшом изменении системы в критической области [11]. Таким образом, между произвольными ячейками одного кластера невозможно оценить степень связности, т. к. она либо присутствует, либо отсутствует. Однако с гидродинамической точки зрения связность между парой ячеек может быть и близкой к нулю, но всё же ненулевой, и близкой к максимальной величине, в этом случае, с точки зрения теории перколяции, связность между такими парами ячеек будет одинаковой (связность присутствует). Вовторых, теория перколяции рассматривает только дискретные системы и в ней не предусмотрено аппарата для анализа геолого-гидродинамических моделей с непрерывным характером куба песчанистости, который может принимать промежуточные между 0 и 1 значения, 0,$15 ; 0,5 ; 0,72$ и т. п.

Другим методом оценки связности моделей является анализ промысловых данных $[3,4,6,7,10]$, который основан на сопоставлении данных работы пар гидродинамически связанных добывающих и нагнетательных скважин. В качестве примера такого анализа можно привести метод MPI (multiwell productivity index) или многоскважинный индекс продуктивности [6], в котором на основе дебитов скважин рассчитывается коэффициент $\lambda$, характеризующий силу связи между каждой парой нагнетательной и добывающей скважин. Свой способ оценки гидродинамической связности предложили С. Тиан и Р. Хорн [10], в котором для каждой пары нагнетательной-добывающей скважин рассчитывается модифицированный коэффициент корреляции дебита-приемистости (1). 


$$
r^{\prime}=-\frac{\sum_{i=1}^{n}\left(q_{i}^{P}-q^{\text {Pref }}\right)\left(q_{i}^{I}-q^{\text {Iref }}\right)}{\sqrt{\sum_{i=1}^{n}\left(q_{i}^{P}-q^{\text {Pref }}\right)^{2}} \sqrt{\sum_{i=1}^{n}\left(q_{i}^{I}-q^{\text {Iref }}\right)^{2}}},
$$

где $r^{`}$ - модифицированный коэффициент корреляции; $i$ - номер пары скважин; $q_{i}^{p}-$ дебит добывающей скважины; $q^{P r e f}-$ опорный дебит добывающей скважины; $q_{i}^{I}-$ приемистость нагнетательной скважины; $q^{\text {Iref }}$ - опорная приемистость добывающей скважины.

Все приведенные выше методы оценки гидродинамической связности также имеют свои недостатки: во-первых, для их использования необходимы промысловые данные хорошего качества [7], а во-вторых, с помощью этих методов невозможно рассчитать связность произвольной модели, т. к. будет необходима реализация определенной системы разработки с известными расстояниями между добывающими и нагнетательными скважинами, от которой напрямую будет зависеть величина связности коллектора.

\section{Определение коэффициента связности коллектора}

Связность коллектора геологической модели в первую очередь определяется тем, как ячейки коллектора расположены друг относительно друга в объеме сеточной области, объединяясь в кластеры, и тем, как эти кластеры взаимосвязаны. При прочих равных условиях величина связности коллектора определяет, какое количество объема дренируется произвольной скважиной и какой у этой скважины будет потенциальный дебит. В этом случае в расчёт не берутся фильтрационно-емкостные характеристики коллектоpa, а основной упор делается на то, как взаимосвязан коллектор в области этой скважины. Исходя из этих соображений, авторы в качестве максимальной связности предлагают использовать дебит скважины на установившемся режиме течения в модели, в которой все ячейки заданы коллектором (единичная песчанистость), и такой дебит называть опорным. При этом коэффициент связности $\left(\mathrm{K}_{\mathrm{c}}\right)$ будет определяться как отношение дебита произвольной скважины или модели к опорному, при равных значениях коэффициента пористости и проницаемости. Для исключения влияния фазовых проницаемостей необходимо рассчитывать дебиты на однофазных моделях

$$
\mathrm{K}_{\mathrm{c}}=\mathrm{Q} / \text { Qref },
$$

где $\mathrm{K}_{\mathrm{c}}$ - коэффициент связности; Q - дебит через модель на установившемся режиме течения; Qref опорный дебит через модель.

Процедура определения коэффициента связности модели будет проводится в несколько этапов. На первом этапе с одной стороны модели все ячейки, прилегающие к торцу, задаются как перфорированные добывающей скважиной. С противоположной стороны модели все ячейки, прилегающие к торцу, задаются как перфорированные нагнетательной скважиной. Далее для исключения влияния неоднородности по фильтрационно-емкостным свойствам (ФЕС) кубы пористости и проницаемости задаются некоей постоянной величиной (например, как среднее значение по модели, но вопрос не принципиальный, т. к. конкретная величина ФЕС, фигурирующия как константа, на значение связности влияния не оказывает). На втором этапе модель загружается в гидродинамический симулятор, между торцами модели задается перепад давления и оценивается дебит добывающей скважины (который будет равен приемистости нагнетательной скважины) на установившемся режиме течения с исследуемым кубом песчанистости и единичным. Финальный коэффициент связности рассчитывается по вышеуказанной формуле (2), и он будет зависеть только от неоднородности распределения коллектора в объеме резервуара (рис. 3). Максимальные значения коэффициента связности будут означать, что перетоки флюида в модели будут максимально возможными, а минимальные значение связности будут означать, что гидродинамическая связь между торцами модели отсутствует и ячейки коллектора формируют разобщенные между собой тела. Промежуточные значения коэффициента связности будут определять значение дебита произвольных скважины в модели.

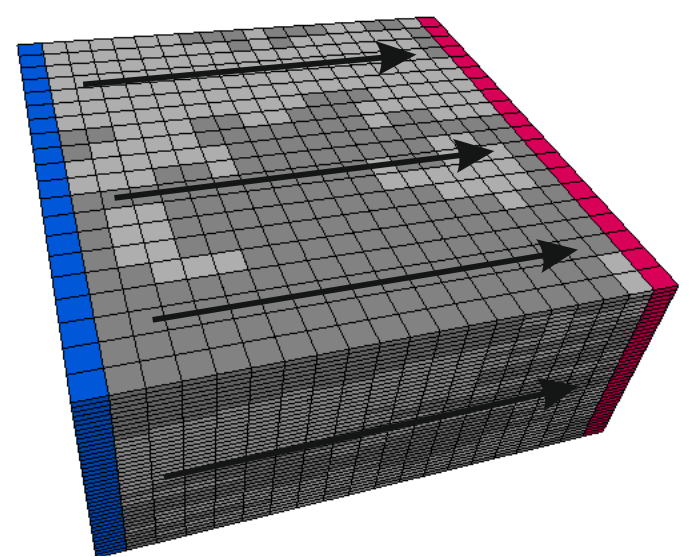

Рис. 3. Схема определения коэффициента связности коллектора. Синие ячейки - задань нагнетательныли, красные - добывающими. При этом градиент давления между этими ячейками сохраняется постоянным. Стрелками показано направление градиента давления. Темные ячейки - коллектор, светлье ячейки - неколлектор

Fig. 3. Estimation of connectivity coefficient scheme. Liquid is injected into blue cells, and produced from red ones. Pressure drop stays constant during the experiment. Arrows point out flow direction. Dark cells are reservoir, light ones are non-reservoir

Для оценки коэффициента связности при разных методах распределения коллектора в геологической модели, а также для изучения влияния неоднородности распределения коллектора была создана синтетическая геологическая модель. Размер модели $10 \times 10$ км, толщина 20 м. Модель была разбита на ячейки размером $100 \times 100 \times 0,5 \mathrm{M}$, таким образом, размерность модели составила $\mathrm{I} \times \mathrm{J} \times \mathrm{K} 100 \times 100 \times 40$ ячеек. Коэффициент пористости во всех ячейках был задан 0,15 д. ед., коэффициент проницаемости 100 мД. На основе этой геологической модели была создана фильтрационная модель со следующими параметрами: 
- коэффициент пористости 0,15 д. ед.;

- коэффициент проницаемости 100 мД;

- фильтрация одной фазы (вода);

- вязкость воды 0,39 сП;

- перепад давления между добывающими и нагнетательными скважинами модели 40 атм.

Для оценки влияния различных эффектов, связанных с геометрией и характером течения в моделях, был проведен ряд расчётов, в которых исследовались режимы течения флюида в модели, влияние детальности и размера модели. Это было необходимо для оценки зависимости коэффициента связности от геометрии модели и, если зависимость есть, разработки методов её учета.

\section{Влияние режима течения на коэффициент связности}

В предложенном методе расчёта коэффициента связности возникает вопрос выбора режима течения флюида в модели. В реальных залежах, разбуренных по равномерной эксплуатационной сетке, поток флюида будет иметь скорее радиальный характер [14], а в способе расчёта, который предлагается, оценивается связность коллектора при линейном режиме течения. Для того чтобы оценить значимость этих различий, на примере построенной синтетической модели были оценены величины коэффициента связности на радиальном и линейном режиме течения при одном распределении коллектора в залежи (рис. 4). Для оценки коэффициента связности при радиальном режиме течения в модели была выбрана пятиточечная система разработки, которая характеризуется соотношением добывающих к нагнетательным скважинам 1:1. Такое же соотношение было предложено в синтетической модели. Коллектор в модели распределялся методом SIS с рангами вариограмм, изменяющимися от 1 до 8 км, с шагом 1 км и песчанистостью, изменяющейся от 0,1 до 0,9. На каждой итерации оценивался коэффициент связности на моделях с радиаль- ным и с линейным режимами течения. Коэффициент корреляции между связностью, рассчитанной по радиальному и линейному режимам течения, составил 0,96, что позволяет сделать заключение о том, что коэффициент связности, рассчитанный по линейному режиму течения, будет обладать репрезентативностью. Плюсами данного подхода является отсутствие необходимости расстановки нагнетательных и добывающих скважин и возможность оценки параметра раздельно по направлениям I и J. Из недостатков следует отметить необходимость соблюдения прямоугольных границ модели. Учет непрямоугольности границ при расчёте линейного коэффициента связности - это тема дальнейших исследований.

\section{Влияние размера модели на коэффициент связности}

Для оценки влияния численной дисперсии гидродинамических симуляторов на величину коэффициента связности [15] был проведен ряд расчётов на синтетических моделях различной размерности, но заведомо большей, чем масштабы неоднородности коллектора. В синтетической модели методом SIS рассчитывалось распределение коллектора, радиус вариограммы задавался небольшой и составлял 500 и 1000 м, для исключения влияния неоднородности распределения коллектора. Неоднородность распределения коллектора оказывает влияние на статистические характеристики модели (средняя песчанистость, ранги экспериментальных вариограмм и т. п.), когда ранги вариограмм превосходят примерно половину размера модели [16]. Таким образом, выбранные ранги, много меньшие размеров модели, гарантированно позволят подобного влияния избежать. Далее исходная модель последовательно обрезалась таким образом, чтобы её размерность в одном направлении изменялась от 3 до 99 с шагом 1, и на каждой итерации рассчитывался коэффициент связности и оценивалось его отклонение от исходного (рис. 5).
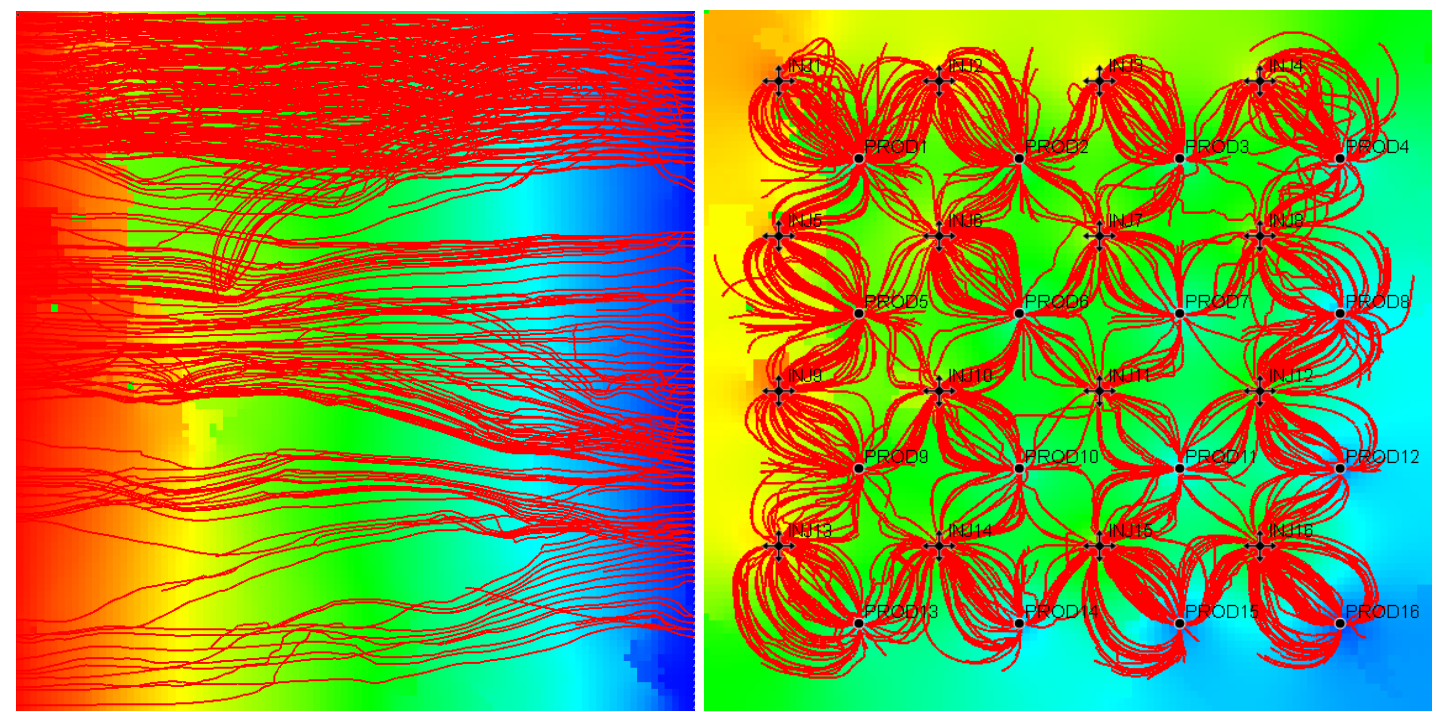

Рис. 4. Линии тока (красным) при оценке коэффициента связности с линейнымм (слева) и радиальным (справа) режимами течения. Цветом обозначено пластовое давление

Fig. 4. Streamlines (red) in models for connectivity coefficient estimation with linear (left) and radial (right) flow regimes. Color means reservoir pressure 


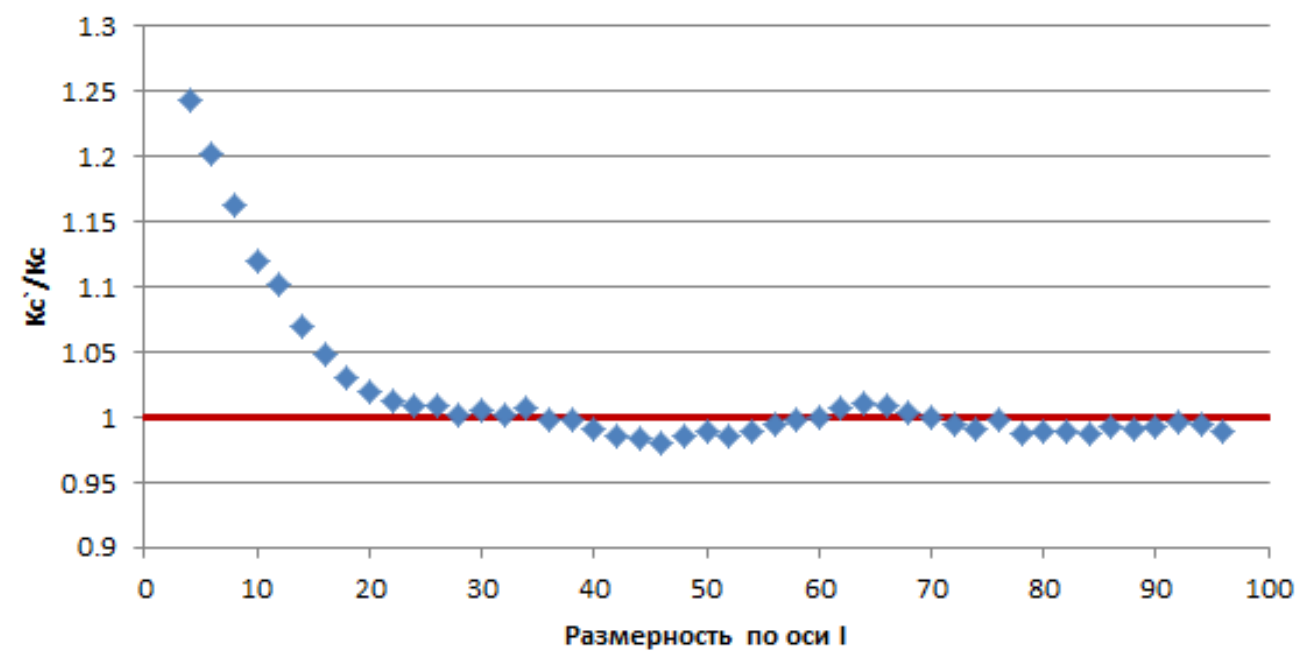

Pис. 5. Типичное отношение коэффициента связности в обрезанной модели (Кс') к коэффициенту связности исходной модели (Кс) в зависимости от размерности модели по оси I

Fig. 5. Typical connectivity coefficient values in cut and source models with relation to model size in I axis

Расчёты показали, что при размерности по оси I модели около 20-25 ячеек связность модели выходит на плато и колеблется в зависимости от того, какая часть тел оказывается отрезанной (рис. 5). Таким образом, эффект масштаба для геологических моделей не будет проявляться при размерности модели более 25 ячеек, при меньших значениях коэффициент связности будет оказываться завышенным.

\section{Влияние размера ячейки на коэффиициент связности}

При оценке связности было сделано предположение, что результат, возможно, будет зависеть от размерности модели, что характерно для многих методов оценки параметров неоднородности геологических моделей [17].

Для оценки влияния эффекта было подготовлено три модели, исходная и две измельченных. Исходная размерностью $100 \times 100$ ячеек, в которой при помощи метода SIS моделировалось распределение коллектор-неколлектор, при этом параметры распределения выбирались следующие: вертикальный ранг состав- лял 2 м, латеральный - 3,5 км, песчанистость изменялась от 10 до $100 \%$ с шагом $10 \%$. Такие параметры в среднем характеризуют залежи, сформированные в прибрежно-морских обстановках осадконакопления, которые являются характерными для большинства терригенных нефтегазоносных залежей Западной Сибири [18]. Пространство между торцами модели измельчалось в 4 и 16 раз при помощи ключевого слова CARFIN, что соответствует размеру ячейки $50 \times 50$ и $25 \times 25$ м.

Расчёты показали, что при одинаковом распределении коллектора результат определения коэффициента связности не зависит от размера ячеек, коэффициенты корреляции между коэффициентом связности, определенном на исходной модели и на измельченной в 4 и 16 раз, составили 1,00 в обоих случаях (рис. 6).

Таким образом, коэффициент связности геологических моделей будет зависеть только от выбранного метода распределения коллектора в модели и его параметров и не будет зависеть от размерности модели и размера ячеек.
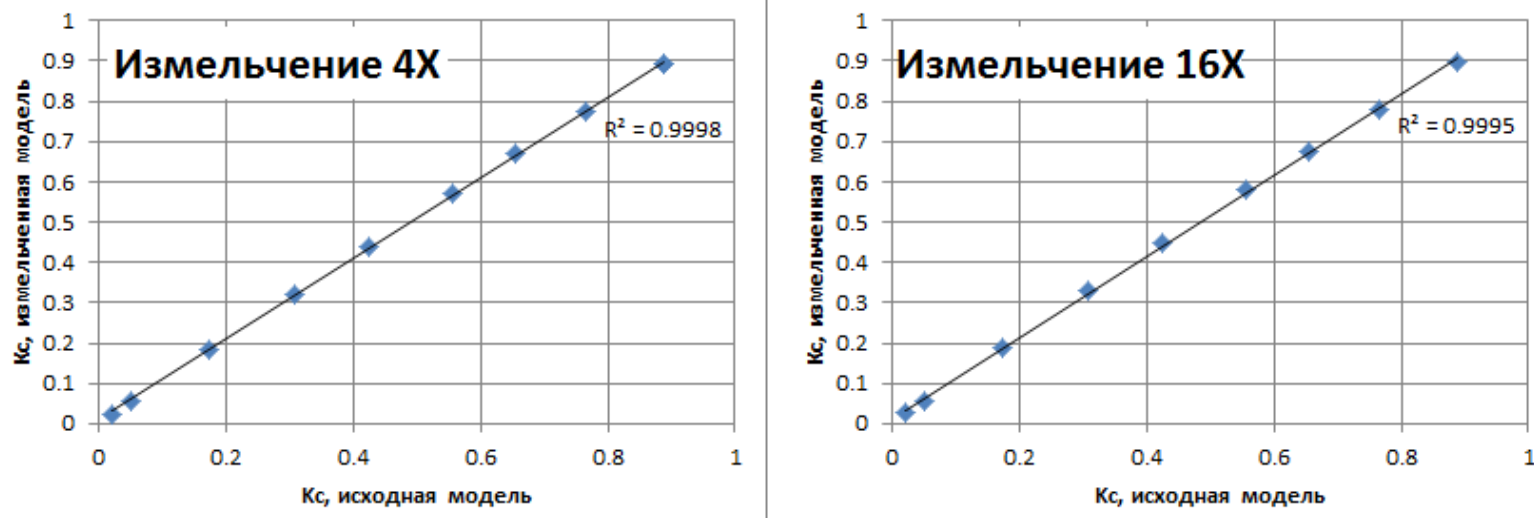

Рис. 6. Сравнение коэффициента связности в исходной и измельченных моделях

Fig. 6. Source and refined model connectivity coefficients comparison 


\section{Характеристика коэффициента связности для различных геологических моделей}

Для понимания величин коэффициента связности и диапазонов их изменения в зависимости от различных параметров распределения и неоднородности коллектора в залежах нефти и газа была создана синтетическая модель, на которой оценивалась величина связности коллектора с разными латеральными и вертикальными радиусами, порогами и анизотропией вариограмм, а также с азимутом анизотропии. Для моделей, не обладающих изометричностью, оценивалась анизотропия коэффициента связности коллектора. На каждой итерации происходило моделирование куба литотипов методом SIS с заданными параметрами, далее расчитывался куб песчанистости, который выгружался и использовался для расчёта коэффициента связности напрямую через гидродинамическую модель. Список изменяемых параметров и диапазон их вариации приведены в табл. 1. Минимум и максимум для каждого параметра определялся значенями, характерными для месторождений нефти и газа $[18,19]$.

Таблица 1. Диапазон и шаг изменения параметров распределения коллектора в модели

Table 1. Parameters variation range and step for reservoir modelling

\begin{tabular}{|l|c|c|}
\hline \multicolumn{1}{|c|}{$\begin{array}{c}\text { Параметр } \\
\text { Рarameter }\end{array}$} & $\begin{array}{c}\text { Диапазон } \\
\text { изменения } \\
\text { Range }\end{array}$ & $\begin{array}{c}\text { Шаг } \\
\text { изменения } \\
\text { Step }\end{array}$ \\
\hline $\begin{array}{l}\text { Главный ранг вариограмы } \\
\text { Main variogram range }\end{array}$ & $1-10$ км/km & 1 км/km \\
\hline $\begin{array}{l}\text { Bторостепенный ранг варио- } \\
\text { граммы } \\
\text { Secondary variogram range }\end{array}$ & $1-10$ км/km & 1 км/km \\
\hline $\begin{array}{l}\text { Aзимут вариограмы } \\
\text { Variogram azimuth }\end{array}$ & $\begin{array}{l}0-90 \text { граду- } \\
\text { сов/degrees }\end{array}$ & $\begin{array}{c}10 \text { граду- } \\
\text { сов/degrees }\end{array}$ \\
\hline $\begin{array}{l}\text { Коэффициент песчанистости } \\
\text { NTG }\end{array}$ & $0,1-0,9$ & 0,1 \\
\hline $\begin{array}{l}\text { Эффект самородков } \\
\text { Nugget effect }\end{array}$ & $0-0,9$ & 0,1 \\
\hline
\end{tabular}

\section{Радиус вариограмм и песчанистость}

Радиус вариограмм и коэффициент песчанистости задают изменчивость распределения коллектора в пространстве и поэтому напрямую влияют на гидродинамическую связность геолого-гидродинамических моделей [5]. Расчёты на синтетических моделях показали, что корреляция между коэффициентом связности и песчанистости достаточно высока и составляет 0,98 , однако при одном значении песчанистости наблюдается хорошая корреляция с латеральным рангом вариограмм (рис. 7).

При объединении обоих зависимостей было получено уравнение регрессии связности геологической модели с песчанистостью и рангом вариограммы (3). Среднеквадратичная ошибка определения коэффициента связности составила менее 0,01 д.ед.

$$
\begin{gathered}
\mathrm{K}_{\mathrm{c}}=1,21 \mathrm{E}-5 \times \mathrm{Lr}+ \\
+\left(6,03 \mathrm{E}-5 \times \mathrm{NTG}^{2}+5,57 \mathrm{E}-3 \times \mathrm{NTG}-0,133\right),
\end{gathered}
$$

где $\mathrm{Kc}$ - коэффициент связности, д.ед; $\mathrm{Lr}$ - латеральный ранг вариограммы, м; NTG - песчанистость модели, д. ед.
Осредненные значения коэффициента связности, полученные на 10 реализациях синтетической модели, сведены в табл. 2.

Таблица 2. Коэффищиенты связности для разных значений песчанистости и рангов вариограмм

Table 2. Connectivity coefficient values for different NTG and variogram ranges

\begin{tabular}{|c|c|c|c|c|}
\hline $\begin{array}{c}\text { Paнг, км/Песчанистость, \% } \\
\text { Range, km/NTG, \% }\end{array}$ & 20 & 40 & 60 & 80 \\
\hline 1 & 0,02 & 0,18 & 0,42 & 0,70 \\
\hline 3 & 0,04 & 0,23 & 0,47 & 0,74 \\
\hline 5 & 0,06 & 0,24 & 0,48 & 0,75 \\
\hline 7 & 0,08 & 0,27 & 0,49 & 0,76 \\
\hline
\end{tabular}

В целом исследования коэффициента связности для моделей с разными коэффициентами песчанистости не подтверждают наличие порогового значения песчанистости, который был установлен в работе Д. Ховадик и Дж. Лару [8]. Это подтверждается приведёнными ниже графиками коэффициента связности для 10 реализаций куба литологии, построенного с радиусом корреляции 3 км. Коэффициент связности во всех случаях плавно увеличивается от 0 до 1 , в то время как степень связности, рассчитанная на основе теории перколяции, будет иметь явно S-образную форму с перегибом, примерно соответствующим точке предела протекания для трехмерных сеток (рис. 8). Такое поведение кривой перколяционной связности может быть объяснено тем, что теория перколяции подразумевает критический характер исследуемых процессов, в которых всегда будет зона нестабильности, выраженная на графике лавинообразным ростом исследуемых величин [15].

\section{Анизотропия вариограмм}

В ходе работ предполагалось, что анизотропия вариограмм может влиять на коэффициент связности за счёт снижения перетоков в поперечно расположенные ячейки коллектора, тем самым препятствуя потоку в направлении главной оси [20]. Для установления степени этого влияния был проведен анализ на синтетической модели, в которой главный ранг вариограммы менялся от 1 до 10 км, для каждого значения главного ранга второстепенный менялся от 1 км и до значения первого ранга. Таким образом, были просчитаны варианты анизотропных вариограмм с отношением первого ранга ко второму от 1 (отсутствие анизотропии) до 10 (вариограмма вытянута в 10 раз). Расчёты показали, что влияние анизотропии на связность не зависит от абсолютной величины рангов вариограмм, а зависит от песчанистости модели и от величины отношения рангов главного ко второстепенному (рис. 9).

\section{Эфрфект самородков}

Увеличение величины эффекта самородков повышает неоднородность геологических моделей, и тем самым приводит к уменьшению связности моделей [20]. Для предельного случая с эффектом самородков, равным 1, распределение коллектора подчиняется 
равномерному закону распределения и самокорреляция будет отсутствовать. Для исследования влияния эффекта самородков были проведены расчёты на синтетической модели с вертикальным рангом 3500 м и песчанистостью 50 \%, в которой эффект самородков изменялся от 0 до 0,9 с шагом 0,1 д. ед. Для каждого значения эффекта самородка было рассчитано 10 равновероятных реализаций.
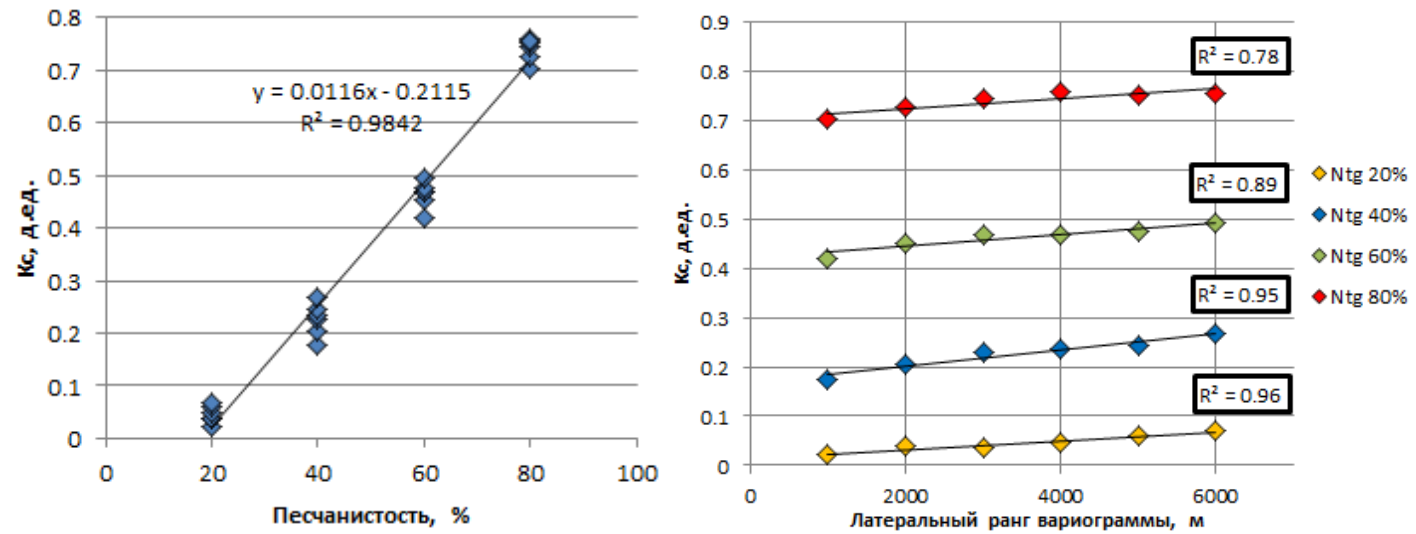

Pис. 7. Коэффициенты корреляции связности с песчанистостью (слева) и с рангом вариограммы (справа)

Fig. 7. Correlation coefficient between connectivity, NTG (right) and variogram range (left)
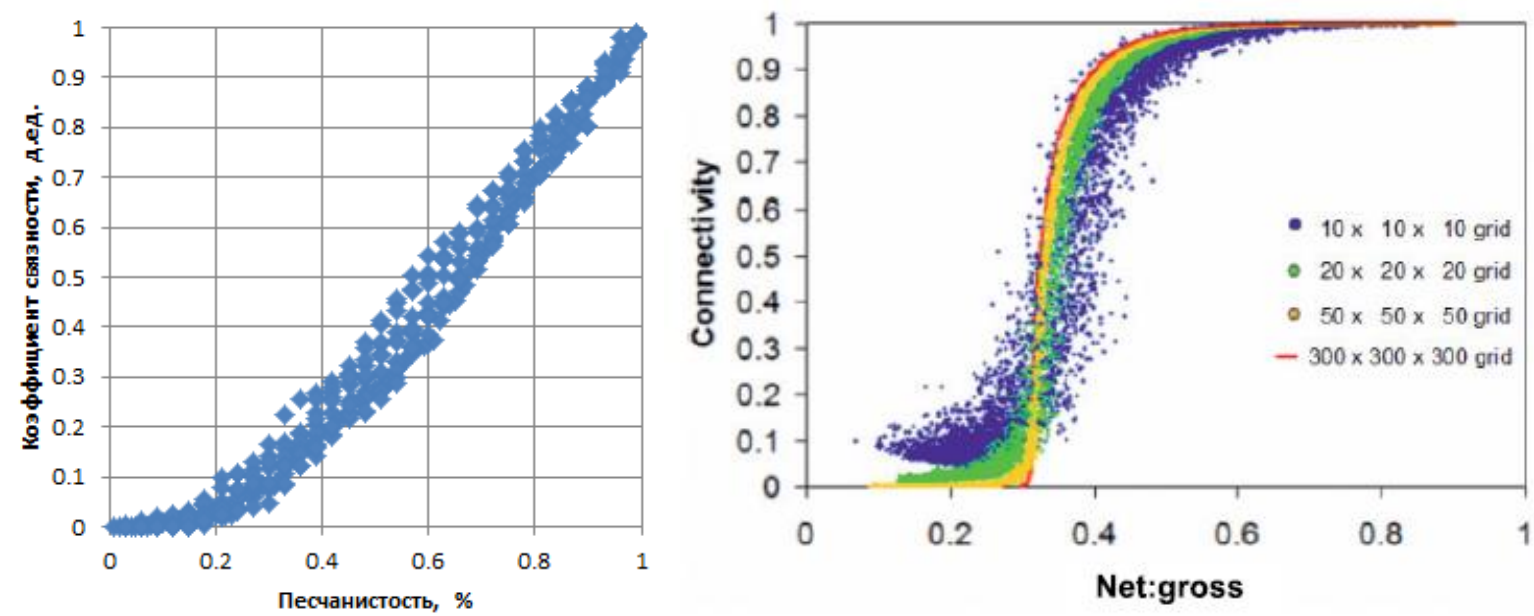

Рис. 8. Зависимость связности от песчанистости, посчитанная по фильтрационной модели (слева) и на основе теории перколяции [8] (справа)

Fig. 8. Connectivity with relation to NTG estimated by simulator (left) and percolation theory methods [8] (right)

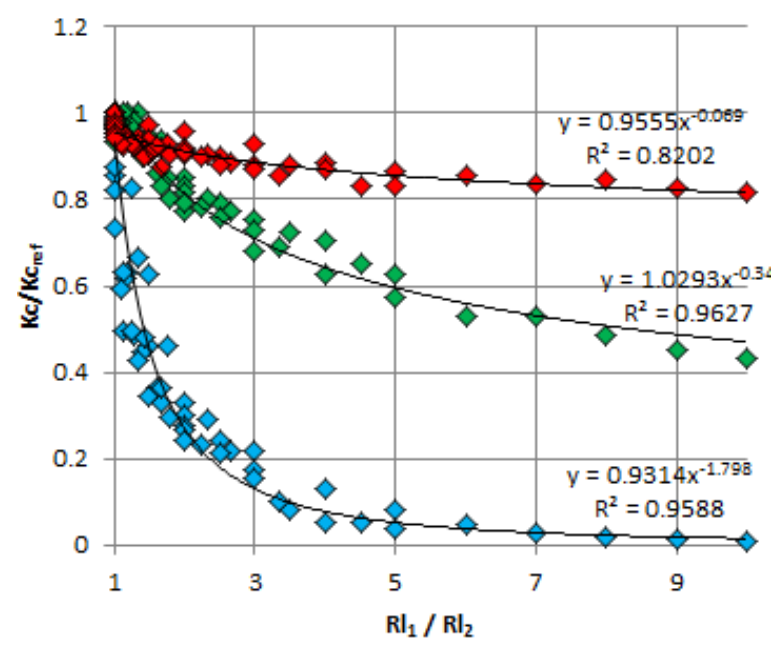

$\diamond N \operatorname{tg} 20 \% \diamond N \operatorname{tg} 50 \% \diamond N \operatorname{tg} 80 \%$
Pис. 9. Зависимость коэффициента связности от анизотропии вариограмм. Фильтрация вдоль направления первой вариограммы. Кс - коэффиичент связности, Kсrеf - коэффициент связности для изотропной вариограммы с данным рангом, Rll - латеральный ранг вариограммы в главном направлении, Rl2 - латеральный ранг вариограммы во второстепенном направлении

Fig. 9. Connectivity coefficient with relation to variogram anisotropy. Flow direction is along main variogram range. Kc-connectivity coefficient, Kcref-connectivity coefficient for isotropic model, Rll - main variogram range, $R l 2$ - secondary variogram range 


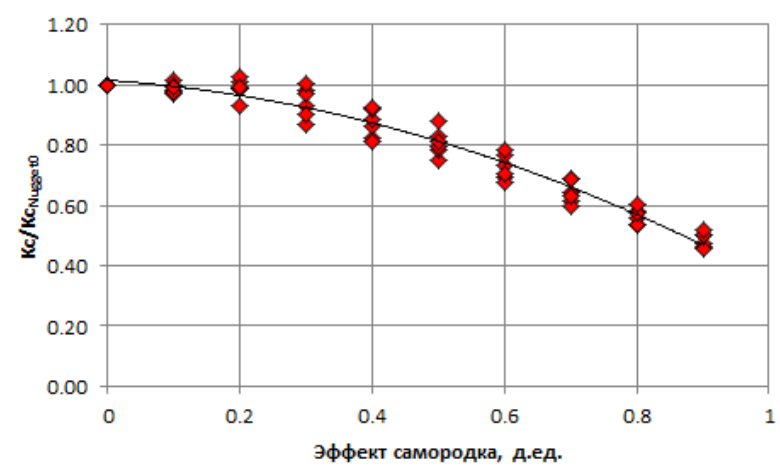

Рис. 10. Зависимость связности коллектора от эффекта самородка

Fig. 10. Connectivity coefficient with relation to nugget effect value

Расчёты показали, что при небольшом значении эффекта самородка, приблизительно до значения 0,2 д. ед., во-первых, не происходит снижения $\mathrm{K}_{\mathrm{c}}$, a во-вторых, возможно даже некоторое его увеличение за счёт появления связанных ячеек между ранее несвязанными телами. В рассчитанных моделях максимальное увеличение связности от повышения эффекта самородка составило 104 \%. Далее связность коллектора монотонно снижается до значения примерно половины связности от исходной.

В дальнейших исследованиях связности геологических моделей планируется разработка методики для оценки коэффициента связности на статических моделях, что с одной стороны позволит экономить время на его оценку и с другой стороны позволит строить карты связности и оценивать этот параметр локально. Также планируется работа по оценке коэффициента связности для различных обстановок осадконакопления и взаимосвязи этого параметра с другими геологическими факторами, влияющими на внутреннее строение залежей нефти и газа.

\section{Выводы}

Предложен количественный способ оценки связности геолого-гидродинамических моделей. Коэффициент связности рассчитывается как отношение дебита через весь объем модели к опорному дебиту и имеет размерность долей единицы или процентов. Опор-

\section{СПИСОК ЛИТЕРАТУРЫ}

1. Новый адаптивный подход к геолого-гидродинамическому моделированию длительно разрабатываемых месторождений и залежей / Е.Н. Тараскин, И.С. Гутман, С.А. Руднев, А.З. Захарян, С.О. Урсегов // Нефтяное хозяйство. - 2017. - № 12. C. $78-83$.

2. Дюбрюль О. Геостатистика в нефтяной геологии. - М.: ИКИ 2009. - $256 \mathrm{c}$.

3. Alabert F.G., Modot V. Stochastic models of reservoir heterogeneity impact on connectivity and average permeabilities. SPE $24893 / / 67^{\text {th }}$ Annual Technical Conference and Exhibition of the SPE. - Washington DC, USA, 1992. - C. 355-370.

4. Budding M.C., Paardekam A.H.M., Van Rossem S.J. 3D connectivity and architecture in sandstone reservoirs. SPE 22342 // SPE International Meeting on Petroleum Engineering. - Beijing, China, 1992. - P. 131-139.

5. Cosentino L. Integrated reservoir studies. - Paris: Editions Technip, 2001. -310 p. ный дебит рассчитывается на исследуемой модели, в которой куб песчанистости во всех ячейках задан единичным. Предложенный параметр более показателен как характеристика неоднородности распределения, чем связность, рассчитанная методами, основанными на теории перколяции, поскольку позволяет учитывать степень связности дифференцировано. В отличие от методов оценки связности, основанных на анализе работы пар нагнетательных и добывающих скважин, предложенный метод позволяет получить оценку неоднородности на залежах без истории разработки и независимо от используемой системы разработки месторождения. Возможно два метода оценки коэффициента связности для модели: на линейном и радиальном режимах течения. Оба показателя тесно взаимосвязаны с коэффициентом корреляции, равным 0,96, при этом оценка коэффициента связности на линейном режиме течения позволяет оценить анизотропию распределения коллектора в различных направлениях. Исследования, проведенные на синтетических моделях, показали, что предложенный способ количественной оценки связности не будет зависеть от геометрии и размерности моделей, а будет зависеть только от взаиморасположения ячеек коллектора, таким образом он оказывается презентативным для любых геолого-гидродинамических моделей, используемых для проектирования разработки залежей.

Для оценки величин коэффициента связности были проведены расчёты на синтетических моделях, в которых распределения коллектора рассчитывалось методом последовательного индикаторного моделирования. Расчёты показали, что наибольшее влияние на коэффициент связности оказывает изменение параметров песчанистости и эффекта самородков. Ранги вариограмм в статистически стационарной геологической модели, напротив, не оказывают значительного влияния на гидродинамическую неоднородность модели.

Предложенный коэффициент связности успешно используется авторами для классификации реализаций куба литотипов при мультивариантном моделировании и для выбора метода построения куба песчанистости при детерминистическом моделировании.

6. Kaviani D., Valko P., Jensen J. Application of the Multiwell productivity index-based method to evaluate interwell connectivity. SPE 129965// SPE Latin American and Caribbean Petroleum Engineering Conference. - Lima, Peru, 2010. URL: https://onepetro.org/ conference-paper/SPE-129965-MS (дата обращения 23.09.2019).

7. Kaviani D., Jensen J. Reliable connectivity evaluation in conventional and heavy oil reservoirs: a case study from Senlac heavy oil pool, Western Saskatchewan. SPE 137504 // Canadian Unconventional Resources \& International Petroleum Conference. - Calgary, Canada, 2010. URL: https://onepetro.org/conference-paper/SPE137504-MS (дата обращения 16.10.2018).

8. Larue D.K., Hovadik J.M. Connectivity of channelized reservoirs: a modelling approach // Petroleum Geoscience. - 2006. - V. 12. P. 291-308.

9. Salomao M.C. Analysis of flow in spatially correlated systems by applying the percolation theory. SPE $39039 / / 5^{\text {th }}$ Latin American and Caribbean Petroleum Engineering Conference and Exhibition. Rio de Janeiro, Brazil, 1997. URL: https://onepetro.org/conferencepaper/SPE-39039-MS (дата обращения 08.10.2018). 
10. Tian C., Horne R.N. Inferring interwell connectivity using production data. SPE-181556-MS // SPE annual Technical Conference and Exhibition. - Dubai, UAE, 2016. URL: https://onepetro.org/conference-paper/SPE-181556-MS (дата обращения 27.08.2019).

11. Кадет В.В. Методы теории перколяции в подземной гидромеханике. - М.: Изд-во ЦентрЛитНефтеГаз, 2008. - 96 с.

12. Эфрос А.Л. Физика и геометрия беспорядка. - М.: Изд-во «Наука», 1982.- $268 \mathrm{c}$.

13. Mashi M., King P.R., Nurafza P.R. Fast estimation of performance parameters in fractured reservoirs using percolation theory. SPE 94186 // SPE Europec/EAGE Annual Conference. - Madrid, Spain, 2005. URL: https://onepetro.org/conference-paper/SPE-94186-MS (дата обращения 08.10.2018).

14. Fanjul J., Vicente M. Reservoir connectivity evaluation and upscaled model screening using streamline simulation. SPE 164312 // SPE Middle East Oil and Gas Show and Conference. - Manama, Bahrain, 2013. URL: https://onepetro.org/conference-paper/SPE164312-MS (дата обращения 02.10.2018).

15. Harpole K., Hearn C. The role of numerical simulation in reservoir management of a West Texas carbonate reservoir. SPE 10022 //
International Petroleum Exhibition and Technical Symposium of the Society of Petroleum Engineers. - Bejing, China, 1982. P. 759-775.

16. Закревский К.Е., Попов В.Л. Вариограмный анализ геологических тел // Экспозиция Нефть Газ. - 2018. - № 1. - С. 10-14.

17. Tang H., Liu N. Static connectivity and heterogeneity analysis and dynamic uncertainty estimation. IPTC 12877 // International Petroleum Technology Conference. - Kuala Lumpur, Malaysia, 2008. URL: https://onepetro.org/conference-paper/IPTC-12877MS (дата обращения 16.10.2018).

18. Закревский К.Е. Об оценке горизонтального радиуса вариограмм // Нефтяное хозяйство. - 2017. - № 11. - Р. 32-33.

19. Reynolds A. Dimensions of paralic sandstone bodies // AAPG Bulletin. - 1999. - V. 83. - № 2. - P. 211-229.

20. Дойч К.В. Геостатистическое моделирование коллекторов. М.; Ижевск: ИКИ, 2011. - 400 с.

\section{Информация об авторах}

Попов В.Л., главный специалист отдела геологического моделирования ОАО «ТомскНИПИнефть».

Поднебесных A.B., кандидат геолого-минералогических наук, эксперт, докторант Уфимского государственного нефтяного технического университета.

Пыльник C.B., кандидат физико-математических наук, главный специалист отдела моделирования ОАО «ТомскНИПИнефть». 
UDC 553.984

\title{
DEFINITION AND DESCRIPTION OF THE OIL AND GAS RESERVOIR CONNECTIVITY COEFFICIENT
}

\author{
Viktor L. Popov', \\ PopovVL@tomsknipi.ru
Alexander V. Podnebesnykh², PodnebesnykhAV@mail.ru \\ Sergey V. Pylnik'1, \\ PopovVL@tomsknipi.ru \\ 1 JSC «TomskNIPIneft», \\ 72, Mira avenue, Tomsk, 634026, Russia. \\ 2 Ufa State Petroleum Technological University, \\ 1, Cosmonavtov street, Ufa, 450062, Russia.
}

The relevance of the research is caused by the need in quantitative evaluation of reservoir connectivity of models for classifying realizations in multivariant modelling and optimization of selection of methods and parameters for reservoir geomodelling.

The main aim of the research is to define connectivity coefficient and estimate its representative validity for different size and geometry models; to evaluate impact of reservoir heterogeneity parameters on connectivity coefficient value.

Objects of the research are geological and simulation models of oil and gas reservoirs.

Methods of the research are geological modelling and reservoir simulation, statistical analysis of the results of geomodeling and reservoir simulation.

Results. The paper briefly describes the existing methods for evaluating the connectivity of oil and gas reservoirs and new approach to connectivity estimation. New connectivity coefficient is defined as a ratio of model liquid rate to unit model liquid rate. Unit model means that net to gross ratio value for all cells is 1 and the model provides maximum possible liquid rate. The paper considers the impact of the model geometry and scale on the connectivity coefficient value. The authors have studied the relation of connectivity coefficient to net to gross ratio, variogram ranges and anisotropy, nugget effect. The connectivity coefficient was stated as a variable, which characterizes the ability of reservoir to pass fluid independent of absolute and relative permeability, porosity, transmissibility etc. The connectivity coefficient as opposed to percolation theory methods can provide continuous evaluation of fluid flow between model cells and does not require valid production history of reservoir.

\section{Key words:}

Geomodel, reservoir connectivity, connectivity coefficient, variogram, nugget effect, sequential indicator simulation, percolation, multiwell productivity index.

\section{REFERENCES}

1. Taraskin E.N., Gutman I.S., Zakharian A.Z., Ursegov S.O. New adaptive approach to geological and hydrodynamic modeling of fields and reservoirs with long production history. Oil industry, 2017, no. 6, pp. 78-83. In Rus.

2. Dubrule O. Geostatistika v neftyanoy geologii [Geostatistics in petroleum geology]. Moscow, IKI Publ., 2009. 256 p.

3. Alabert F.G., Modot V. Stochastic models of reservoir heterogeneity: impact on connectivity and average permeabilities. SPE 24893. 67 $7^{\text {th }}$ Annual Technical Conference and Exhibition of the SPE. Washington DC, USA. 1992. pp. 355-370.

4. Budding M.C., Paardekam A.H.M., Van Rossem S.J. 3D connectivity and architecture in sandstone reservoirs. SPE 22342. SPE International Meeting on Petroleum Engineering. Beijing, China. 1992. pp. 131-139.

5. Cosentino L. Integrated reservoir studies. Paris, Editions Technip, 2001. $310 \mathrm{p}$.

6. Kaviani D., Valko P., Jensen J. Application of the Multiwel productivity index-based method to evaluate interwell connectivity. SPE 129965. SPE Latin American and Caribbean Petroleum Engineering Conference. Lima, Peru, 2010. Available at: https://onepetro.org/conference-paper/SPE-129965-MS (accessed 23 August 2019).

7. Kaviani D., Jensen J. Reliable connectivity evaluation in conventional and heavy oil reservoirs: a case study from Senlac heavy oil pool, Western Saskatchewan. SPE 137504. Canadian Unconventional Resources \& International Petroleum Conference. Calgary, Canada, 2010. Available at: https://onepetro.org/conference- paper/SPE-137504-MS (accessed 16 October 2018).
8. Larue D.K., Hovadik J.M. Connectivity of channelized reservoirs: a modelling approach. Petroleum Geoscience, 2006, vol. 12, pp. 291-308.

9. Salomao M.C. Analysis of flow in spatially correlated systems by applying the percolation theory. SPE 39039. $5^{\text {th }}$ Latin American and Caribbean Petroleum Engineering Conference and Exhibition. Rio de Janeiro, Brazil, 1997. Available at: https://onepetro.org/conference-paper/SPE-39039-MS (accessed 8 October 2018)

10. Tian C., Horne R.N. Inferring interwell connectivity using production data. SPE-181556-MS. SPE annual Technical Conference and Exhibition. Dubai, UAE, 2016. Available at: https://onepetro.org/conference-paper/SPE-181556-MS (accessed 27 August 2019)

11. Kadet V.V. Metody teorii perkolyatsii v podzemnoy gidromehan$i k e$ [Percolation theory methods in underground hydromechanics] Moscow, TsentrLitNefteGaz Publ., 2008. 96 p.

12. Efros A.L. Fizika i geometriya besporyadka [Disorder physics and geometry]. Moscow, Nauka Publ., 1982. 268 p.

13. Mashi M., King P.R., Nurafza P.R. Fast estimation of performance parameters in fractured reservoirs using percolation theory. SPE 94186. SPE Europec/EAGE Annual Conference, Madrid, Spain, 2005. Available at: https://onepetro.org/conferencepaper/SPE-94186-MS (accessed 8 October 2018).

14. Fanjul J., Vicente M. Reservoir connectivity evaluation and upscaled model screening using streamline simulation. SPE 164312. SPE Middle East Oil and Gas Show and Conference. Manama, Bahrain, 2013. Available at: https://onepetro.org /conference-paper/SPE-164312-MS (accessed 2 October 2018). 
15. Harpole K., Hearn C. The role of numerical simulation in reservoir management of a West Texas carbonate reservoir. SPE 10022. International Petroleum Exhibition and Technical Symposium of the Society of Petroleum Engineers. Beijing, China, 1982. pp. 759-775.

16. Zakrevskiy K.E., Popov V.L. Variogram analysis of geological bodies. Ekspozitsia Neft Gas, 2018, no. 1, pp. 10-14. In Rus.

17. Tang H., Liu N. Static Connectivity and Heterogeneity Analisis and Dynamic uncertainty estimation. IPTC 12877 // International Petroleum Technology Conference. Kuala Lumpur, Malaysia,

2008. Available at: https://onepetro.org/conference-paper/IPTC12877-MS (дата обращения 16.10.2018).

18. Zakrevskiy K.E. On the assessment of lateral range of the variograms. Oil industry, 2017, no. 11, pp. 32-33. In Rus.

19. Reynolds A., Dimensions of paralic sandstone bodies. AAPG Bulletin, 1999, vol. 83, no. 2, pp. 211-229.

20. Deutsch C.V. Geostatisticheskoe modelirovanie kollektorov [Geostatical reservoir modelling]. Moscow, Izhevsk, IKI Publ., 2011. $400 \mathrm{p}$.

Received: 14 July 2019.

Information about the authors

Viktor L. Popov, senior specialist, JSC «TomskNIPIneft».

Alexander V. Podnebesnykh, Cand. Sc., expert, Ufa State Petroleum Technological University.

Sergey V. Pylnik, Cand. Sc., senior specialist, JSC «TomskNIPIneft». 